American Journal of Applied Sciences 9 (6): 846-850, 2012

ISSN 1546-9239

(C) 2012 Science Publications

\title{
The Mathematical Model of Power System with Static Var Compensator in Long Transmission Line
}

\author{
Prechanon Kumkratug \\ Department of Electrical Engineering, Faculty of Engineering at Si Racha, \\ Kasetsart University, 199 M.6, Tungsukhla, Si Racha, Chonburi, 20230, Thailand
}

\begin{abstract}
Problem statement: It is becoming increasingly important to fully utilize the existing transmission system assets due to environmental legislation, rights-of-way issues and costs of construction and deregulation policies that introduced in recent years. The Static Var Compensator (SVC) has been proposed for the better control power flow and dynamic performance. The exact long transmission line model consists of the resistance and reactance. Most of previous researches studies transient stability performance of the SVC in SMIB System with neglecting resistance of the line. Thus the fully capability of the SVC on transient stability improvement of power system may not be applied. The consideration of the resistance causes in the difficulty of deriving mathematical model. Approach: This study investigates the effect of the SVC on transient stability of the power system with consideration the exact long transmission line mode. The concept of two-port network is applied to simplify the mathematical model of the power system. The proposed method is tested on sample system and compared on various cases. Results: The first swing of rotor angle curve of the faulted system without resistance is obviously higher than that of with resistance whereas the second swing of the faulted system without resistance is slightly less than that of with resistance. The system with the SVC can improve transient stability of power system. Conclusion: It is found from this study that the SVC and resistance of the line can improve first swing of rotor angle. The resistance of the line provides the negative effect on second swing of rotor angle. The simulation results indicate that for practical long line, the resistance is very import parameters for evaluating transient stability of power system.
\end{abstract}

Key words: Static Var Compensator (SVC), Critical Clearing Time (CCT), Single Machine Infinite Bus (SMIB), transient stability, increasingly important

\section{INTRODUCTION}

Nowadays, the demand of electricity has dramatically increased and a modern power system becomes a complex network of transmission lines interconnecting the generating stations to the major loads points in the overall power system in order to support the high demand of consumers. It is becoming increasingly important to fully utilize the existing transmission system assets due to environmental legislation, rights-of-way issues and costs of construction and deregulation policies that introduced in recent years. A number of Flexible AC Transmission System (FACTS) controllers, based on the rapid development of power electronics technology, have been proposed for better utilization of the existing transmission systems (Nabhan and Abdallah, 2010; Bawazir and Rahman, 2010; Osuwa and Igwiro, 2010; Zarate-Minano et al., 2010; Padma and Rajaram, 2011).

The Static Var Compensator (SVC) is the shunt FACTS devices. It consists of the capacitor bank reactor bank and thyristor as shown in Fig. 1. The thyristors control the reactance that dictates the power flow through a line. The SVC can be applied for improving transient stability of power system (Arun and Krishnan, 2011).

The evaluation of Critical Clearing Time (CCT) of power system is one of the most important research areas for power engineers because it indicates the robustness of the faulted power system. The rotor angle of the synchronous generator determines the stability of power system. Although the stability of the synchronous machine is used to represent the stability of the power system, all of the power system components such as transmission line and transformer affect the stability of the power system.

The transmission line is one of the most important parts in power system components. Most of the fault occurs at the transmission line. It is generally divided into three major categories; short, medium and long model whose distance are about $80 \mathrm{~km}^{-1}$, above $80-250$ and above $250 \mathrm{~km}^{-1}$, respectively. Many previous researches used simple transmission line model by neglecting its resistance or capacitance. To fully utilization the existing system, the exact transmission line should be further investigated. 
Am. J. Applied Sci., 9 (6): 846-850, 2012

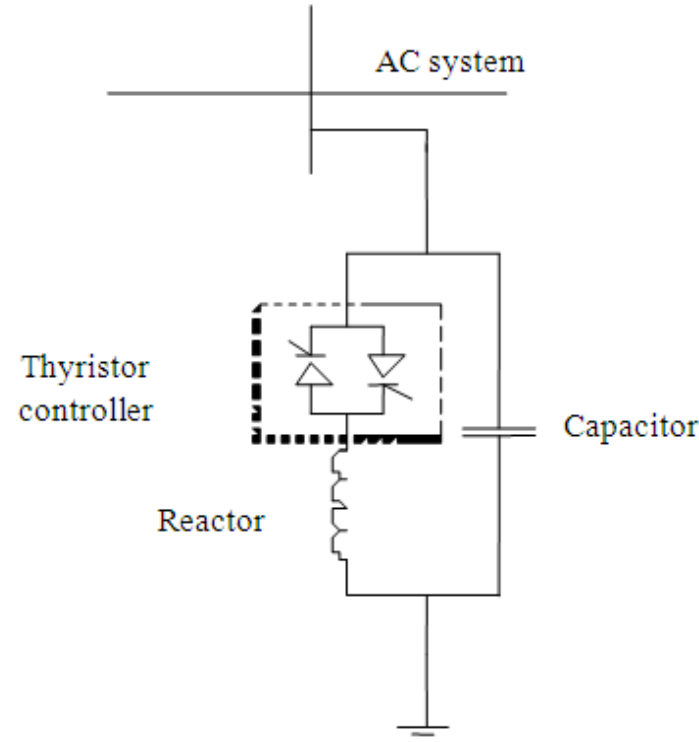

Fig. 1: Schematic diagram of SVC

This study will investigate the capability of the SVC on transient stability of the SMIB system with the exact long transmission line model. The concept of two-port network is applied to simplify the mathematical model of the power system. The sample system consisting the practical long transmission line is used to investigate in this study. The proposed method is tested on various cases.

\section{MATERIALS AND METHODS}

Mathematical model: The transmission line is considered as long line when its distance is beyond 150 $\mathrm{km}$. The basic model of long transmission line consists of the lump of medium transmission line model with serial models as shown in Fig. 2a. Its simpler model is represented by a model as shown in Fig. $2 b$.

TheZ' and Y'are:

$\mathrm{Z}^{\prime}=\mathrm{Z}_{\mathrm{c}} \sinh (\gamma)$

$\mathrm{Y}^{\prime}=2 / \mathrm{Z}_{\mathrm{c}} \tanh (\gamma)$

$\gamma=\sqrt{\mathrm{ZY}} \operatorname{andZ}_{\mathrm{c}}=\sqrt{\frac{\mathrm{Z}}{\mathrm{Y}}}$

Figure 3a shows the single line diagram of power system consisting of a generator, a transformer, four long transmission lines and SVC. Figure $3 b$ shows the equivalent of Fig. 3a. The generator is represented by a synchronous voltage in quadrature axis $\left(\mathrm{E}_{\mathrm{q}}{ }_{\mathrm{q}}\right)$ behind direct transient reactance $\left(\mathrm{X}_{\mathrm{d}}{ }_{\mathrm{d}}\right)$. The $\mathrm{V}_{\mathrm{b}}$ is the voltage at infinite bus.

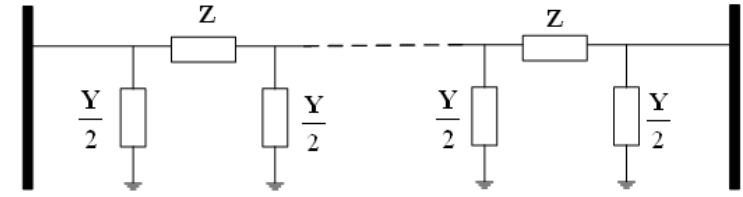

(a)

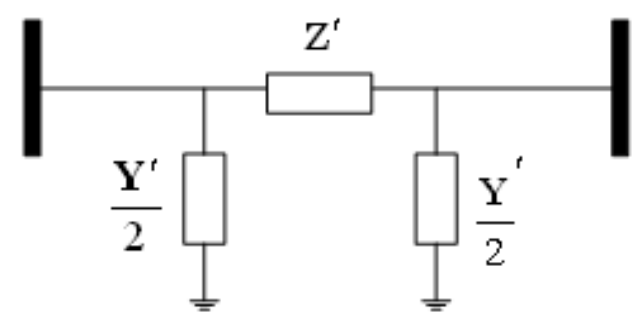

(b)

Fig. 2: Long transmission line model (a) Basic model with the serial $\pi$ models (b) a $\pi$ model

The exact long transmission line model is represented by the impedance $\left(\mathrm{Z}_{\mathrm{L}}\right)$ which consists of a resistance $\left(\mathrm{R}_{\mathrm{L}}\right)$ and reactance $\left(\mathrm{X}_{\mathrm{L}}\right)$. The SVC can be modeled as the shunt variable susceptance $\left(\mathrm{B}_{\mathrm{SvC}}\right)$ as shown in Fig. $3 \mathrm{~b}$. This study will apply the concept of the two-port network to simplify the equivalent in Fig. 3b. Each component of power system and a SVC can be represented the matrix of two-port networks $(\mathrm{A}, \mathrm{B}, \mathrm{C}$ and D) as shown in Fig. 3c and given by Eq. 3-14:

$$
\begin{aligned}
& \mathrm{A}_{1}=\mathrm{A}_{2}=\mathrm{A}_{\mathrm{SVC}}=1 \\
& \mathrm{~A}_{3}=\mathrm{A}_{4}=\mathrm{A}_{5}=\mathrm{A}_{6}=\cosh (\gamma) \\
& \mathrm{B}_{1}=\mathrm{j} \mathrm{X}_{\mathrm{d}}^{\prime} \\
& \mathrm{B}_{2}=\mathrm{j} \mathrm{X}_{\mathrm{t}} \\
& \mathrm{B}_{3}=\mathrm{B}_{4}=\mathrm{B}_{5}=\mathrm{B}_{6}=\mathrm{Z}_{\mathrm{c}} \sinh (\gamma) \\
& \mathrm{B}_{\mathrm{SVC}}=0 \\
& \mathrm{C}_{1}=\mathrm{C}_{2}=0 \\
& \mathrm{C}_{3}=\mathrm{C}_{4}=\mathrm{C}_{5}=\mathrm{C}_{6}=\frac{1}{\mathrm{Z}_{\mathrm{c}}} \sinh (\gamma) \\
& \mathrm{C}_{\mathrm{SVC}}=\mathrm{jB}_{\mathrm{SVC}} \\
& \mathrm{D}_{1}=\mathrm{D}_{2}=0
\end{aligned}
$$


Am. J. Applied Sci., 9 (6): 846-850, 2012

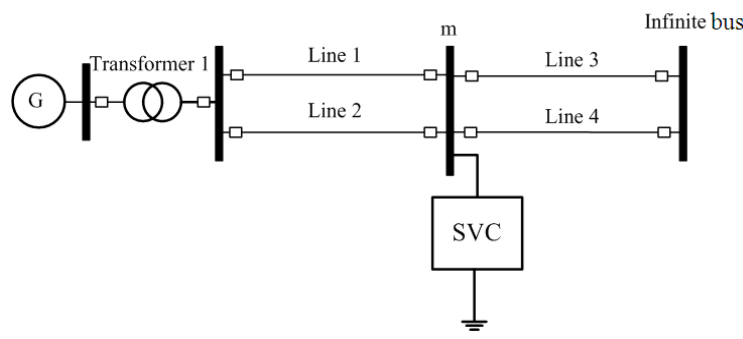

(a)

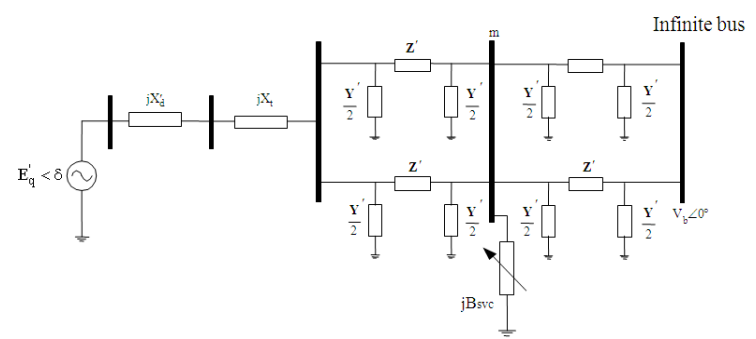

(b)

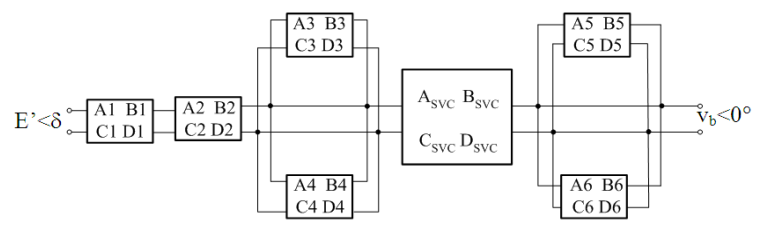

(c)

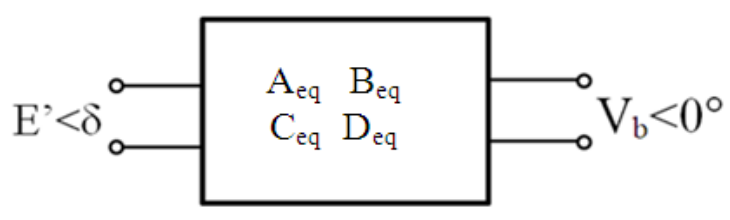

(d)

Fig. 3: Single machine infinite bus system with a SVC (a) schematic diagram (b) equivalent circuit (c) two -port networks diagram (d) the net two-port network

$\mathrm{D}_{3}=\mathrm{D}_{4}=\mathrm{D}_{5}=\mathrm{D}_{6}=\cosh (\gamma)$

$\mathrm{D}_{\mathrm{SVC}}=1$

It can be seen from the Fig. 3c that some ports are in series and in shunt connection. For example, a port 1 and port 2 are in series connection whereas port 3 and port 4 are in shunt connection. Thus with the series combination of port 1 and port 2, a new port is given by Eq. 15-18:

$\mathrm{A}_{\mathrm{s}}=\mathrm{A}_{1} \mathrm{~A}_{2}+\mathrm{B}_{1} \mathrm{C}_{2}$
$\mathrm{B}_{\mathrm{s}}=\mathrm{A}_{1} \mathrm{~B}_{2}+\mathrm{B}_{1} \mathrm{D}_{2}$

$\mathrm{C}_{\mathrm{s}}=\mathrm{A}_{2} \mathrm{C}_{1}+\mathrm{C}_{2} \mathrm{D}_{1}$

$\mathrm{D}_{\mathrm{s}}=\mathrm{B}_{2} \mathrm{C}_{1}+\mathrm{D}_{1} \mathrm{D}_{2}$

Similarly, with the shunt combination of port 3 and port 4, a new port is given by Eq. 19-22:

$A_{\text {sh }}=\left(A_{3} B_{4}+A_{4} B_{3}\right) /\left(B_{3}+B_{4}\right)$

$\mathrm{B}_{\mathrm{sh}}=\mathrm{B}_{3} \mathrm{~B}_{4} /\left(\mathrm{B}_{3}+\mathrm{B}_{4}\right)$

$\mathrm{C}_{\mathrm{sh}}=\mathrm{C}_{3}+\mathrm{C}_{4}+\left(\mathrm{A}_{3}-\mathrm{A}_{4}\right)\left(\mathrm{D}_{4}-\mathrm{D}_{3}\right) /\left(\mathrm{B}_{3}+\mathrm{B}_{4}\right)$

$\mathrm{D}_{\mathrm{sh}}=\left(\mathrm{B}_{4} \mathrm{D}_{3}+\mathrm{B}_{3} \mathrm{D}_{4}\right) /\left(\mathrm{B}_{3}+\mathrm{B}_{4}\right)$

With the above concepts, the net two-port network diagram is shown in Fig. $3 d$. Here $A_{e q}, B_{e q}, C_{e q}$ and $D_{e q}$ are the element in net matrix of net two-port networks.

The output electrical power of synchronous machine $\left(\mathrm{P}_{\mathrm{e}}\right)$ is given by Eq. 23:

$P_{e}=\frac{A_{e q}\left(E_{q}^{\prime}\right)^{2}}{B_{\text {eq }}} \cos \left(\theta_{\text {Beq }}-\theta_{\text {Aeq }}\right)-\frac{V_{b} E_{q}^{\prime}}{B_{\text {eq }}} \cos \left(\theta_{\text {Beq }}+\delta\right)$

Here:

$\mathrm{A}_{\text {eq }}=\mathrm{A}_{\text {eq }} \angle \theta_{\text {Aeq }} \mathrm{B}_{\text {eq }}=\mathrm{B}_{\text {eq }} \angle \theta_{\text {Beq }}$

The dynamic equation for evaluating critical clearing time of the system in Fig. 3a is given by:

$\dot{\delta}=\omega$

$\dot{\omega}=\frac{1}{\mathrm{M}}\left[\mathrm{P}_{\mathrm{m}}-\mathrm{P}_{\mathrm{e}}\right]$

Here, $\delta, \omega$ and $\mathrm{P}_{\mathrm{m}}$ as given in Eq. 24-25 are the rotor angle, speed, mechanical input power and moment of inertia, respectively of synchronous machine. The $\mathrm{P}_{\mathrm{e}}$ is the output electrical power of synchronous as given in Eq. 23.

It can be mentioned here that the shunt variable susceptance of the SVC as given in Eq. 8 is changed during the dynamic state for improve the transient stability. This study uses the linear control given by:

$\mathrm{B}_{\mathrm{SVC}}=\mathrm{K} \omega$

Here $\mathrm{K}$ in Eq. 26 is the constant gain control. 


\section{RESULTS}

The proposed method is tested on the sample system consider the diagram of sample system is shown in Fig. 2a. The system data are:

Generator: $\mathrm{H}=5, \mathrm{Xt}=0.1 \mathrm{pu}, \mathrm{X}^{\prime} \mathrm{d}=0.20 \mathrm{pu}, \mathrm{E}^{\prime} \mathrm{q}=$ $1.22<31.64 \mathrm{pu}$.

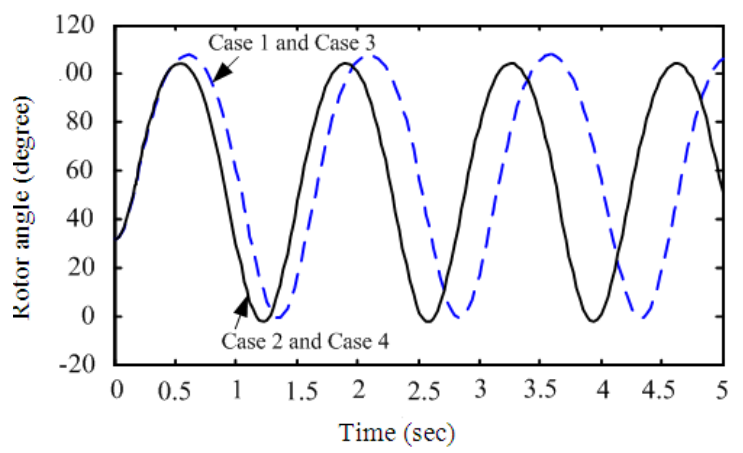

Fig. 4: Swing curve of the system without FACTS devices for $\mathrm{tcl}=170 \mathrm{~m} \mathrm{sec}$

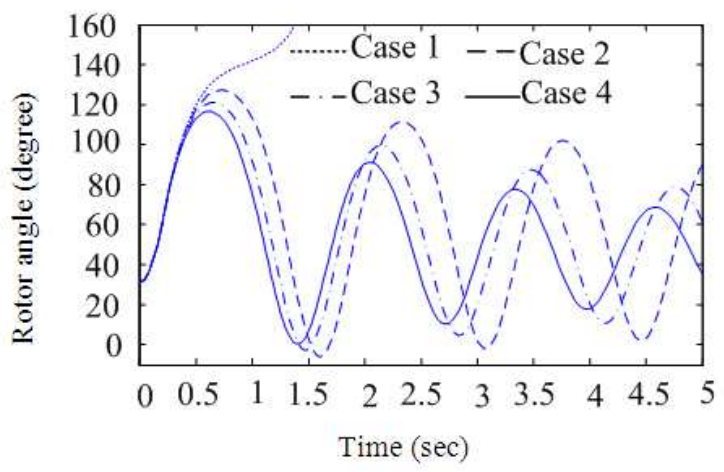

Fig. 5: Rotor angle of the system with various gains of a $\mathrm{SVC}$ for $\mathrm{t}_{\mathrm{cl}}=190 \mathrm{~m} \mathrm{sec}$

Table 1: The maximum and minimum rotor angle of the system without SVC for $\mathrm{tcl}=170 \mathrm{~m} \mathrm{sec}$

\begin{tabular}{llllr}
\hline Case & $\mathrm{R}$ & $\mathrm{Bc}$ & $\delta_{\max }($ degree $)$ & $\delta_{\text {mix }}($ degree $)$ \\
\hline 1 & 0.0000 & 0.000000 & 107.80 & -0.63 \\
2 & 0.0263 & 0.000000 & 105.85 & -1.42 \\
3 & 0.0000 & 0.000594 & 107.80 & -0.63 \\
4 & 0.0263 & 0.000594 & 105.85 & -1.42 \\
\hline
\end{tabular}

Table 2: The maximum and minimum rotor angle of the system without SVC for tcl $=198 \mathrm{~m} \mathrm{sec}$

\begin{tabular}{lrcc}
\hline Case & $\mathrm{K}$ & $\delta_{\max }($ degree $)$ & $\delta_{\operatorname{mix}}($ degree $)$ \\
\hline 1 & 0 & - & - \\
2 & 10 & 127.21 & -6.02 \\
3 & 20 & 121.51 & -2.66 \\
4 & 30 & 116.65 & 0.36 \\
\hline
\end{tabular}

Transmission line data: Voltage level $500 \mathrm{kV}, 300 \mathrm{~km}$, $\mathrm{f}=50 \mathrm{~Hz}, \mathrm{R}=0.016 \Omega / \mathrm{km}, \mathrm{L}=0.97 \mathrm{mH} / \mathrm{km}, \mathrm{C}=$ $0.0115 \mu \mathrm{f} / \mathrm{km}^{-1}$

The series $Z^{\prime}$ and the shunt admittance $Y^{\prime}$ of the long line at fundamental frequency are $4.6426+\mathrm{j} 89.92$ and $0+\mathrm{j} 0.00109$, respectively. The percentage of $\mathrm{R} / \mathrm{X}$ and $\mathrm{B}_{\mathrm{c}} / \mathrm{X}$ of the line are $=5.26 \%$ and $\mathrm{B}_{\mathrm{c}} / \mathrm{X}=$ $0.001188 \%$, respectively. With the given reactance of the line $(\mathrm{X})=0.5 \mathrm{pu}$, the per unit of $\mathrm{R}$ and $\mathrm{B}_{\mathrm{c}}$ are 0.0263 and $0.00059 \mathrm{pu}$, respective.

It is considered that three phase fault appears at line 2 near bus $\mathrm{m}$ and the fault is cleared by opening circuit breakers at the end of the line. Figure 4 shows the rotor angle of the system without a SVC $(\mathrm{K}=0)$ for the clearing time of fault $\left(\mathrm{t}_{\mathrm{cl}}\right) 170 \mathrm{~m}$ sec. Table 1 summarizes the maximum and minimum rotor angles $\left(\delta_{\text {max }}, \delta_{\text {mix }}\right)$ of Fig. 3.

Figure 5 shows the rotor angle of the system with various gains of a SVC and with consideration of the exact long transmission line model. Here the $t_{c l}$ is increased to $198 \mathrm{msec}$. Table 2 summarizes the maximum and minimum rotor angles of Fig. 5.

\section{DISCUSSION}

It can be seen from the Fig. 4 and Table 1 that resistance of the long line provides the improvement of the first swing stability but not for the second swing. Without the resistance of the long line, the maximum and the minimum rotor angle are 107.80 and $2.44^{\circ}$ respectively whereas with the resistance, the maximum and the minimum rotor angle are 105.85 degree and $1.42^{\circ}$, respectively. In practical long line, the resistance is very import parameters to determine the critical clearing time of the system whereas shunt capacitance doesn't affect on the damping performance because in practical lone line the shunt capacitance is very small. The critical clearing time of the system with exact long transmission line and without FACTS is around 197198 msec. As can be seen in Fig. 5 and Table 2, the system without SVC $(\mathrm{K}=0)$ is considered as unstable. However, with the SVC the system can be considered as stable and the damping performance is improved as the gain is inacreased.

\section{CONCLUSION}

This study investigated the effects of the Static Var Compensator (SVC) on transient stability of the Single Machine Infinite Bus (SMIB) with the consideration of the exact long transmission line model. The concept of two-port network is applied to simplify the mathematical model of the system. The reactance of generator, transformer exact long line model and SVC can be represented by a net two-port network. 
It was found from the simulation results that the resistance of the line provides the improvement of the first swing but not for the second swing. It was found from this study that for practical long line, the resistance is very import parameters to determine the critical clearing time of the single machine infinite system whereas shunt capacitance would not affect on critical clearing time of single machine infinite bus system. It was also found in this study that the SVC can enhance transient stability of power system.

\section{REFERENCES}

Arun, M. and A. Krishnan, 2011. Comparative power analysis of precomputation based content addressable memory. J. Comput. Sci., 7: 471-474. DOI: $10.3844 /$ jcssp. 2011.471 .474

Bawazir, H.M.S. and A.A. Rahman, 2010. A tabu search method for finding minimal multihomogeneous bEzout number. J. Math. Stat., 6: 105-109. DOI: 10.3844/jmssp.2010.105.109
Nabhan, I. and M. Abdallah, 2010. A novel low-power CMOS operational amplifier with high slew rate and high common-mode rejection ratio. Am. J. Eng. Applied Sci., 3: 189-192. DOI: 10.3844/ajeassp.2010.189.192

Osuwa, J.C. and E.C. Igwiro, 2010. Uninterruptible power supply using solar rechargeable battery. Physics. Int., 1: 77-82. DOI: 10.3844/pisp.2010.77.82

Padma, S. and M. Rajaram, 2011. Fuzzy logic controller for static synchronous series compensator with energy storage system for transient stability analysis. J. Comput. Sci., 7: 859864. DOI: $10.3844 /$ jcssp.2011.859.864

Zarate-Minano, R., T. Van Custsem, F. Milano and A.J. Conejo, 2010. Sexuring transient stability using time domain simulations within an optimal power flow. IEEE Trans. Power Syst., 5: 243-253, DOI: 10.1109/TPWRS.2009.203069 